\title{
Environmental Topography and Postural Control Demands Shape Aging-Associated Decrements in Spatial Navigation Performance
}

\author{
Martin Lövdén and Michael Schellenbach \\ Max Planck Institute for Human Development
}

\author{
Barbara Grossman-Hutter and Antonio Krüger \\ Saarland University
}

Ulman Lindenberger

Max Planck Institute for Human Development

\begin{abstract}
This study tests the hypothesis that aging-induced cognitive permeation of sensorimotor functions contributes to adult age differences in spatial navigation performance. Virtual maze-like museums were projected in front of a treadmill. Sixteen 20-30-year-old men and sixteen 60-70-year-old men performed a way-finding task in city-block or variable topographies while walking with or without support. Walking support attenuated age-related decrements in navigational learning. Navigation load increased trunkangle variability for older adults only. Age differences in spatial knowledge persisted despite perfect place-finding performance. City-block topography was easier than variable topography for younger adults only, indicating age-related differences in reliance on spatial relational learning. Attempts at supporting older adults' navigation performance should consider sensorimotor/cognitive interactions and qualitative differences in navigational activity.
\end{abstract}

Keywords: spatial navigation, spatial memory, aging, sensorimotor functioning

Navigation in large-scale environments demands decisions about which way to take based on current goals, perceptual cues, and internal representations that evolve during navigation activity. Learning to navigate to a place in the environment involves acquiring accessible internal representations that can support navigation-a process dependent on multiple cognitive processes such as attentional control, spatial and relational computations in working memory; and the different processes involved in encoding, storing, and retrieving long-term memories. The evolving representation of an environment may contain different landmark positions that are represented in terms of their spatial relationships to one another (i.e., a "cognitive map"; Tolman, 1948; O’Keefe \& Nadel, 1978). Though the existence of mechanisms uniquely devoted to spatial relational coding is debated (e.g., Cohen \& Eichenbaum, 1993; O'Keefe, 1999; Eichenbaum, Dudchenko,

Martin Lövdén, Michael Schellenbach, and Ulman Lindenberger, Center for Lifespan Psychology, Max Planck Institute for Human Development, Berlin, Germany; Barbara Grossman-Hutter and Antonio Krüger, Department of Computer Science, Saarland University.

Antonio Krüger is now at the Institute for Geoinformatics, University of Münster, Münster, Germany.

This work was supported by Deutsche Forschungsgemeinschaft Grant SFB 378 (“Aging, Resources, and Cognition" project) to Ulman Lindenberger. We thank Viktor Müller, Timo von Oertzen, and Hubert Zimmer for helpful discussions; Karl Kapitza for assistance in preparing the study; and Susan Hänig, Hannah Kerstner, Lea Meyer, Daniela Neininger, and Benjamin Straube for their help in collecting the data.

Correspondence concerning this article should be addressed to Martin Lövdén, Center for Lifespan Psychology, Max Planck Institute for Human Development, Lentzeallée 94, D-14195, Berlin, Germany. E-mail: loevden@mpib-berlin.mpg.de
Wood, Shapiro, \& Tanila, 1999), there is general agreement that relational learning applied to the spatial domain forms a flexible basis for navigation in complex environments (e.g., Burgess, Maquire, \& O'Keefe, 2002). In this study, we use the term spatial navigational learning to denote this type of mental activity. In contrast, other forms of navigational activities are relatively deprived of spatial processes and may involve verbal left/right seriallearning or stimulus-response-stimulus chains leading to the goal (i.e., route learning/following; e.g., Hartley, Maguire, Spiers, \& Burgess, 2003). In terms of neuroanatomical underpinnings, neuropsychological and neuroimaging studies have highlighted the role played by the hippocampus, parahippocampal gyrus, parietal lobe, and prefrontal cortex during spatial navigation (see Aguirre \& D'Esposito, 1999; Burgess et al., 2002, for reviews) and the role played by the caudate nucleus for route following (e.g., Bohbot, Iaria, \& Petrides, 2004; Hartley et al., 2003).

Adult age-related performance decrements are pronounced for tasks that require processing components critically contributing to navigation performance. For example, adult age differences are pronounced when demands on relational processes in working memory (Chalfonte \& Johnson, 1996), attentional control (McDowd \& Shaw, 2000), and conjunctive binding of item-item or item-context information are high (Jacoby, 1999; NavehBenjamin, Hussain, Guez, \& Bar-on, 2003; Spencer \& Raz, 1995; for a computational model, see S.-C. Li, Naveh-Benjamin, \& Lindenberger, in press). In the visuospatial domain, age-related performance differences are ubiquitous for mental rotation and visualization tasks (Hertzog \& Rypma, 1991; Kirasic, 2000; Salthouse \& Mitchell, 1989) and for tasks taxing memory for the location of objects (Light \& Zelinski, 1983; Uttl \& Graf, 1993). Moreover, age differences in these types of tasks are more palpable than in comparable verbal tasks (Jenkins, Myerson, Joerding, \& 
Hale, 2000). Assessment of route learning skills provides support for substantial age-related deficits (Lipman, 1991; Wilkniss, Jones, Karol, Gold, \& Manning, 1997). Similarly marked deficits are found for tasks requiring spatial inferences of direction relationships and distances between locations in, for example, supermarkets (Kirasic, 1991, 2000). Finally, clinically important impairments in navigation skills (Klein et al., 1999) and changes in visuospatial test-performance (e.g., Lövdén, Bergman, Adolfsson, Lindenberger, \& Nilsson, in press) are apparent in early stages of dementia. Taken together, age-related differences in various aspects of cognition supporting navigational skills are pronounced.

Though performance decrements during adulthood in various aspects of spatial cognition are well substantiated, little is known about the extent, nature, and attenuation of older adults' deficiencies in complex navigational place learning. One reason for this state of affairs might be that the bulk of research on spatial cognition has either utilized tasks that are relatively remote to real world navigation or examined route learning skills under more ecologically valid but less well controlled conditions. To study the essentials of navigation in complex environments (e.g., building up representations over time through active exploration, detailed and multiple perceptual cues) while maintaining control of the environment, more recent endeavors have used desktop virtual environment (VE) paradigms to study, for example, interindividual differences in navigation performance (e.g., Moffat, Hampson, \& Hatzipantelis, 1998; Moffat, Zonderman, \& Resnick, 2001; Sandstrom, Kaufman, \& Huettel, 1998) and the neurophysiological and neuroanatomical substrates of navigation behavior (e.g., Ekstrom et al., 2003; Grön, Wunderlich, Spitzer, Tomczak, \& Riepe, 2000; Hartley et al., 2003; Janzen \& van Turennout, 2004; Maguire et al., 1998). For example, Moffat et al., (2001) administered a VE maze-learning task involving five learning trials to younger (ages $22-45$ years), middle-aged (45-65), and older (65-91) adults. The VE consisted of interconnected hallways, some leading to the designated goal and others to dead ends. Older adults took longer time to solve each learning trial and traversed a longer distance than younger adults. This study, and similar studies with other VE tasks (e.g., computer-generated Morris Water Maze; e.g., Laurance et al., 2002), confirm pronounced adult age differences in navigation performance with a relatively high degree of experimental control while providing human analogs of well-established animal models (e.g., Morris, 1981; cf. Tolman, 1948).

However, the question arises as to what extent navigation in VEs simulates real world navigation and tax similar neurocognitive mechanisms. Most relevant studies suggest that spatial knowledge acquired in a VE transfers rather well to subsequent navigation in the real world (e.g., Ruddle, Payne, \& Jones, 1997; Witmer, Bailey, Knerr, \& Parsons, 1996), at least for navigation in spatially simpler environments (Richardson, Montello, \& Hegarty, 1999), but there are also important limitations with VE paradigms (see Peruch \& Gaunet, 1998, for overview). Clearly, one of the greatest drawbacks of desktop VE paradigms is that they do not require actual movement through space. For example, Waller, Loomis, and Haun (2004) had three groups of participants to learn locations along an $840 \mathrm{~m}$ long route. One group walked the route and the other two groups watched different videos of the route. Walking during learning significantly reduced pointing error. In the animal model, lesions of the vestibular system impair spatial learning in the radial-arm maze task (Ossenkopp \& Hargreaves, 1993) and reversible inactivation of the vestibular system disrupt "place cell" (O'Keefe \& Dostrovsky, 1971) firing (Stackman, Clark, \& Taube, 2002). These findings indicate that vestibular signals influence the acquisition and expression of hippocampal spatial representations. McNaughton et al. (1996) hypothesized that self-motion establishes a metric for the "cognitive map" and that accurate navigation depends on monitoring both internal self-motion cues and landmarks. Thus, vestibular and proprioceptive cues as well as motor efference copy provide important information for spatial representations.

Though self-motion is an important component of navigational place finding, several studies have indicated that cognitive processes and sensorimotor functions may compete for shared mental resources (see K. Z. H. Li \& Lindenberger, 2002; Woollacott \& Shumway-Cook, 2002, for reviews). For example, Lindenberger, Marsiske, and Baltes (2000) trained younger (ages 20-30 years), middle-aged (40-50), and older adults $(60-70)$ to criterion in memorizing word-lists with the method-of-loci and to walk fast and accurately on two tracks of different complexity. Older adults showed greater reductions in performance under dual-task conditions relative to younger adults (see also Chen et al., 1996). K. Z. H. Li, Lindenberger, Freund, and Baltes (2001) replicated and extended these findings with individualized levels of task difficulty and a more challenging motor task. Older adults maintained high levels of walking performance under dual-task conditions but showed pronounced decrements in the cognitive domain. Clinically oriented observations corroborate these findings (Lundin-Olsson, Nyberg, \& Gustafson, 1997, 1998). For example, Lundin-Olsson et al., (1997) found a significant association between stopping to walk while taking part in a conversation and the frequency of falls within a 6-months follow-up period. The attention-demanding nature of gait in older age generalizes to maintaining a stable upright stance when balance is challenged (e.g., Brauer, Woollacott, \& Shumway-Cook, 2001; Brown, Shumway-Cook, \& Woollacott, 1999; Teasdale, Bard, LaRue, \& Fleury, 1993). For example, Brown et al., (1999) examined recovery of balance before and after perturbations on a moving platform. In a dual-task condition, participants were required to concurrently perform a counting backward task. Younger and older adults had similar counting speed before perturbations, but the older adults were differentially slowed during the recovery phase. Thus, with advancing adult age sensorimotor functions decline (see Ketcham \& Stelmach, 2001, for review), causing sensorimotor tasks such as walking to impose greater demands on declining cognitive resources.

In this study, we examine the developmental quandary between increasing control demands of sensorimotor functioning and decreasing efficiency of relevant control operations (Lindenberger et al., 2000) in the domain of spatial navigation. To this end, we designed a VE maze-learning paradigm with a walking component. In this paradigm, a VE, designed to give participants the impression of walking through a museum of art, is projected in front of a treadmill. The movement of the treadmill is synchronized to the visual flow of the VE such that participants have the impression of actually walking through the virtual environment. The task for participants is to find and remember the way from the entrance to the bistro. When participants are able to walk the shortest route from the entrance to the bistro twice in a row, the task is completed. Between each learning trial a survey perspective 
of the building is displayed and the participant is asked to place the museum's paintings at the correct spatial positions (see Figure 1). This task served to measure acquired configural knowledge of the spatial relations among the most important landmarks (i.e., the paintings). During performing the task participants wore ultra-

A

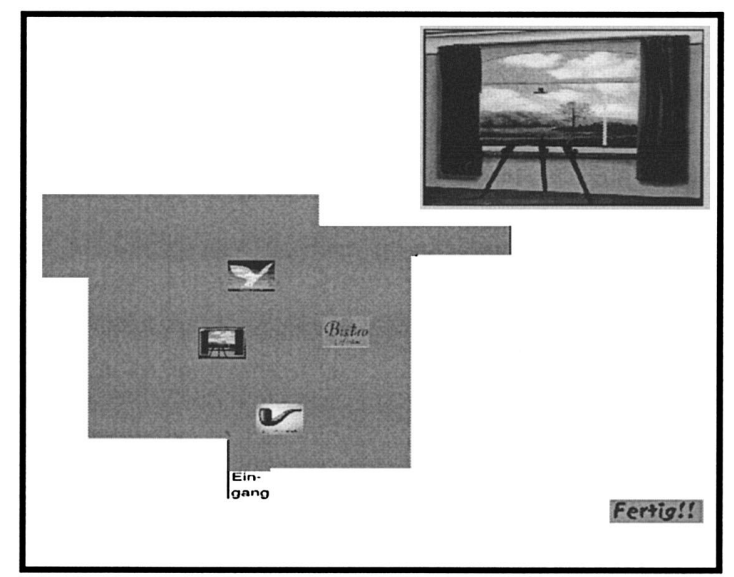

B

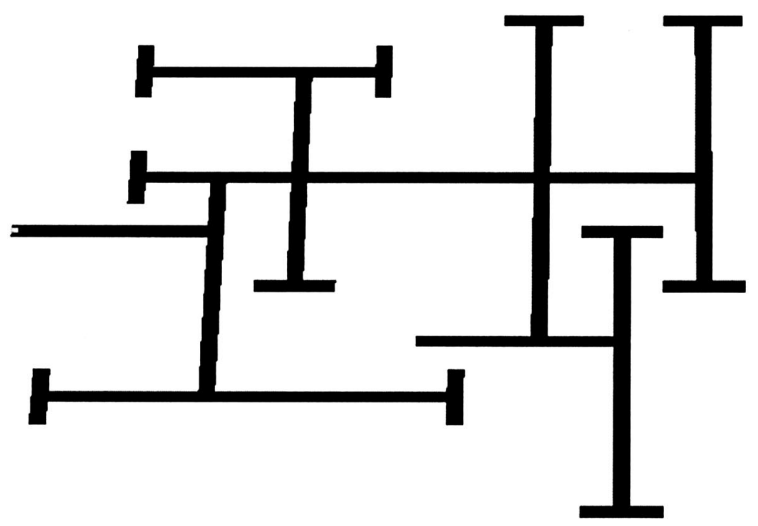

C

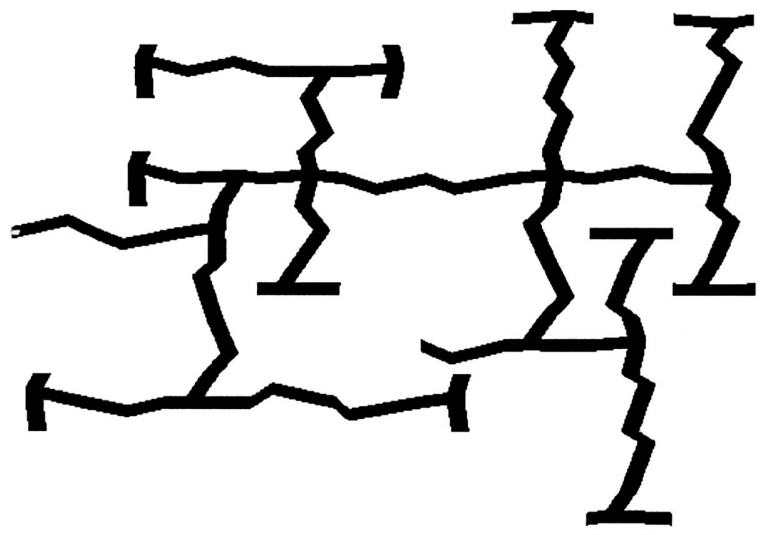

Figure 1. A: The survey view as displayed to the participant in the landmark test (originals were in color). The paintings could be freely dragged and dropped using the mouse. The large painting on display is the currently selected painting. A similar picture, but without the paintings, was displayed before the start of the first trial. B: The city-block topography. C: The variable topography. These views were not shown to participants. sound senders providing data relevant for assessing walking behavior. Because upper body measures might be especially sensitive to age-related reductions in walking stability (e.g., McGibbon \& Krebs, 2001) we focused on trunk-angle variability as an indicator of walking behavior during navigation.

We implemented two experimental factors in the design: maze topography and walking demand. The background for the topography factor stemmed from findings suggestive of interindividual differences (e.g., Moffat et al., 1998) in the use of route learning contra spatial navigational learning as cognitive means for navigation, and, most importantly, from research on age-related differences within animal models. In addition to age-related performance differences linked to associative binding deficits and loss of synaptic connectivity in the hippocampus (e.g., Barnes, Rao, Foster, \& McNaughton, 1992; Smith, Adams, Gallagher, Morrison, \& Rapp, 2000), qualitative age differences in processes supporting navigation behavior have been demonstrated as well. For example, Rapp, Rosenberg, and Gallagher (1987) showed that senescent rats do not acquire spatial information to the same extent as younger rats after training to find a visible platform in the Morris water maze (Morris, 1981), as evidenced by older rats poorer performance in finding a submerged platform after training (see also Barnes, Nadel, \& Honig, 1980; Tanila, Sipilä, Shapiro, \& Eichenbaum, 1997). Thus, aged organisms might preferentially use nonspatial processes to locate places in space whenever such strategies are adaptive whereas younger organisms might rely more on spatial navigational learning. To address this issue in humans, we varied the topography of the maze-like museums by either implementing corridors that were straight or winding. At the same time, the number of decision points, the perceptual cues available at decision points, and large-scale spatial relations among the entrance, bistro, landmarks, and intersections were kept constant. The two resulting versions of a museum, city-block and variable, are depicted from a survey perspective in Figure 1. We expected an effect of topography on navigation performance only to the extent that the cognitive operations in the maze involved spatial navigational learning. Specifically, we expected that the frequent shifts in heading in the variable topography would require continuous updating of the spatial relations among key points in the museums and thus make keeping track of these relations more difficult. On the other hand, the cognitive demands posed on route learning (e.g., verbal left/right serial learning) were constant across versions. In line with findings from the animal model, we predicted an age group by topography interaction, signaling less effect of topography for the older relative to the younger age group.

For the walking demand factor, we varied whether participants were provided with walking support during navigation (holding on to a handrail) or not (not allowed to hold on to the handrail). We chose this implementation of walking demand because previous studies have indicated that supporting gait by holding onto support, such as a handrail, is a preferred and natural way of stabilizing posture in challenging conditions (e.g., K. Z. H. Li et al., 2001). In addition, this manipulation has clear face validity and ecological relevance by mimicking everyday situations (e.g., grabbing the handrail in a bus) as well as assistive aids supporting walking stability (e.g., walking canes). We expected that sensorimotor demands would modulate age differences in navigational place learning. Specifically, we predicted that cognitive permeation of sensorimotor functions in old age would lead to an age group by 
walking demand interaction, signaling disproportionately beneficial effects of walking support on navigation performance for older adults.

\section{Method}

\section{Participants}

Participants were 16 younger $\left(M_{\text {age }}=24.7\right.$ years; $S D_{\text {age }}=3.0$; age range: $20-30)$ and 16 older men $\left(M_{\text {age }}=65.4\right.$ years; $S D_{\text {age }}=3.0$; age range: $60-69)$. They were recruited from undergraduate classes at Saarland University and the volunteer database at the School of Psychology, Saarland University. To be eligible for the study, participants had to report absence of colorblindness, diagnosed balance sicknesses, history of severe dizziness, heart problems, stroke, Parkinson's disease, and present or previous treatment for psychiatric illness. All participants had normal or corrected to normal vision. They received course credits or were paid 72 Euro each.

To achieve the effective sample, seven older adults had to be replaced. Three participants were not able to complete all six sessions of the study for personal reasons and four participants were replaced for not reaching performance criterion in one of the sessions involving the maze-learning tasks (see Procedures). The attrition is causing positive selection biases for the older age group and therefore operates against our hypotheses.

To document cognitive typicality of the sample, Table 1 summarizes the scores on four unit-weighted composites representing performance on tests of visuospatial ability (mental rotations and figure folding), perceptual speed (digit letter and identical pictures), verbal memory (memory-for-text and paired associates), and verbal knowledge (vocabulary and spot-a-word) as a function of age group. The mental rotations and figure folding tests were taken from Vandenberg and Kuse (1978) and designed for this study with the typical versions as models, respectively. Detailed descriptions of the perceptual speed, verbal memory, and verbal knowledge measures are provided in Lindenberger, Kliegl, and Mayr (1993; see also Lövdén, Ghisletta, \& Lindenberger, 2004). Each composite was scaled according to the T-metric $(M=50 ; S D=10)$. An inspection of Table 1 suggests better performance for younger relative to older adults on the visuospatial, perceptual speed, and verbal memory composites, whereas verbal knowledge was inferior among younger adults. Univariate one-way (age group) analyses of variance (ANOVAs) for each composite confirmed these observations: visuospatial, $F(1,30)=24.60, M S E=56.77, p<.001, \eta^{2}=$ .45 ; perceptual speed, $F(1,30)=42.78, M S E=42.60, p<.001, \eta^{2}=.59$; verbal memory, $F(1,30)=10.67, M S E=76.23, p<.01, \eta^{2}=.26$; and verbal knowledge, $F(1,30)=12.38, M S E=73.16, p<.001, \eta^{2}=.29$. Thus, the typical developmental pattern of age-related decrease of fluid abilities and age-related increase (or maintenance) of crystallized abilities was observed (see Lövdén \& Lindenberger, 2005, for review). We con-

Table 1

Cognitive Characteristics as a Function of Age Group

\begin{tabular}{|c|c|c|c|c|}
\hline \multirow[b]{2}{*}{ Variable } & \multicolumn{2}{|c|}{ Younger } & \multicolumn{2}{|c|}{ Older } \\
\hline & $M$ & $S D$ & $M$ & $S D$ \\
\hline Visuo-spatial ability & 55.6 & 4.5 & 43.4 & 9.7 \\
\hline Perceptual speed & 57.5 & 6.4 & 42.5 & 6.7 \\
\hline Verbal memory & 55.0 & 9.0 & 45.0 & 8.8 \\
\hline Verbal knowledge & 44.7 & 11.1 & 55.3 & 4.8 \\
\hline
\end{tabular}

Note. Visuo-spatial ability $=T$-scaled unit-weighted composite of Vandenberg-Kuse mental rotations test (S. G. Vandenberg \& A. R. Kuse, 1978) and a figure folding test; Perceptual speed = identical pictures and digit letters; Verbal memory $=$ memory for - text and paired associates; Verbal knowledge $=$ spot a word and vocabulary. clude that the sample constitutes a satisfactory approximation of the population trends in cognitive functioning.

\section{Materials}

Virtual environment. Five maze-like topographies, one practice maze and four experimental mazes were constructed. The maze topographies were randomly generated within the following constraints: (a) Each maze consisted of five decision points on the direct route from the start to the goal. Three decision points were two-choice alternatives and two were three-choice alternatives; (b) the maze began and ended with a two-choice decision point; (c) the same decision (e.g., right) on the direct route from the start to goal was only allowed to occur twice in a row, regardless of whether intermixed with a straight ahead decision or not; (d) of the total of seven possible incorrect choices, three led directly to a dead end and four to a two-choice decision point before reaching a dead end. The dead ends were constructed so that they were indistinguishable from a normal decision point before a navigational decision was made at that point; (e) all mazes were composed of 22 corridors of which six were of a designated short length, eight were of a middle length, and eight of a long length; and (f) the corridors on the correct way from start to the goal were of equal total length across generated mazes but otherwise randomly assigned with the constraint of not causing overlap among corridors.

Each maze existed in a city-block (straight corridors and 90 degree turns at decision points) and in a variable topography (winding corridors and variable degrees of turning at decision points). Figure 1 illustrates these formats from a survey perspective (never shown to participants). The variable topography was constructed by randomly moving several center points in the corresponding city-block version. The lengths of the corridors were scaled in order to get comparable walking distances.

Five different virtual museums of art were constructed, corresponding to the five different maze-like topographies described above. In a practice maze, an exhibition of Klee paintings was held. The four experimental mazes contained either paintings of van Gogh, Magritte, Breughel, or Warhol. Three paintings were placed on intersections (i.e., decision points) on the shortest route from the start to the goal. The start was symbolized with a sign "Entrance to the (e.g.) Klee museum." The goal was symbolized with a door and a "bistro" sign. The first, third, and fourth intersections had a painting on display. At every intersection in a museum, unique objects that varied in texture and shape within a museum and in category across museums (chairs, ashtrays, trash bins, vases, and stools) were placed. These two types of "landmarks" (objects and paintings) provided unique large-scale positional information and were only visible in relatively close proximity of the intersections. On every corridor in the mazes, self-portraits of the artist holding the exhibition were displayed and various other repetitive objects appeared (e.g., benches, lamps, and no smoking signs). The repetitive nature of these objects gave them little or no largescale positional information value. The texture of the floor varied across museums.

The virtual-reality environment was controlled by an Apple dual $1 \mathrm{GHz}$ Power PC G4 computer, running a $\mathrm{C}++$ simulation software that we designed and programmed. The scenery was projected by means of a SHARP PG-A 10X projector with a rate of 40 frames per second and a resolution of $1024 \times 768$ pixels. The $105 \times 84$-cm projection area allowed for approximately 39 degrees horizontal and 31 degrees vertical field of view when the participant was positioned in the normal walking position, approximately $150 \mathrm{~cm}$ in front of the screen. The software stored the position of the viewpoint at a rate of $30 \mathrm{~Hz}$, as well as all actions performed by the participant.

Interface. Operating two buttons controlled navigation in the virtual environment. While walking without handrail support, the customdesigned button-boxes were handheld (one in each hand). While walking with support, the buttons were attached to the handrail. The handrail resembled normal handlebars and was constructed so that participants could hold on to the handles while pressing the buttons with their thumbs 
(resembling changing gears on a mountain-bike). The handrail was positioned approximately $100 \mathrm{~cm}$ above the walking surface and in front of the participant. Five commands were allowed: take right (right button click), take left (left button), take straight ahead (both buttons at the same time), turn around 180 degrees via left (double click left button), and turn around via right (double click right button). Turning around was allowed in all parts of the maze. The other options were only active in close proximity of intersections. When participants approached an intersection, arrows appeared on the screen to symbolize that the buttons were active and that a decision was required. Response errors (e.g., clicking right when wanting to go left) were very rare and, if anything, likely to be slightly more frequent in the condition of walking with support. Thus, any differences in response errors between the conditions operate against our hypotheses.

The movement of the walking area of the treadmill (a normal exercise treadmill; Woodway GmbH, Weil am Rhein, Germany) was coupled with the virtual environment in the sense that the treadmill smoothly decelerated if the participant approached an intersection without having made a navigational decision and came to a complete stop if no decision was made before reaching the intersection. At the same time, well enough time was available for decisions before the treadmill started to decelerate. In the case the treadmill came to a complete stop, participant remained standing with a view of the intersection including the available navigational choices, paintings, and objects until a decision was made and the movement accelerated to the maximum walking speed of $3.0 \mathrm{~km} / \mathrm{hr}$. This maximum speed was fixed across all participants and conditions. The choice of maximum speed was informed mainly by piloting, but also by previous studies (e.g., Prince, Carriveau, Hébert, \& Winter, 1997), indicating preferred walking velocities around $3.0 \mathrm{~km} / \mathrm{hr}$ for most healthy older $(60-70$ years old) individuals. Walking speed was not under participants' control, with the exception that participants could defer making a decision when they approached an intersection and thus slow down or even come to a complete stop. Pilot studies had established a ratio of the visual flow to the speed of walking that gave rise to a subjective impression of a match. When the treadmill decelerated or accelerated at intersections the perceived visual flow thus changed in a completely synchronized (real-time) manner with the movement. The walking area of the treadmill was $150 \mathrm{~cm}$ long and $43 \mathrm{~cm}$ wide.

Posture recording. Posture during walking was recorded with the ZEBRIS CMS20S ultrasound system (ZEBRIS Medizintechnik GmbH, Isny, Germany). Eight senders attached to the back of participants transmitted ultrasound signals at a rate of $30 \mathrm{~Hz}$ to a receiver positioned 150 $200 \mathrm{~cm}$ away from the normal position of the participant. The $\mathrm{x}, \mathrm{y}$, and $\mathrm{z}$ positions of each sender were fed into a PC and the ZEBRIS WinData (version 2.1955) software. Two senders (one left and one right) were attached to the Achilles tendon directly above the level of the inner foot ankle; two were placed in the hollow of the knee; two at the back of the hip (dorso lateral iliac crest); and two at the shoulder blade positions (median edge of scapulas) when arms were in resting position.

Maze-learning task. Before starting the task, a survey perspective of the museum, displaying the outer walls, the position of entrance, and the position of bistro, appeared for 15 seconds (see Figure 1). Next, a firstperson view of the entrance to the museum was displayed and the participant started walking in the museum after pressing any of the two buttons. The task for the participant was to find and remember the way to the museum's bistro.

Upon arrival at the bistro, the survey perspective appeared again and the participant was asked to place miniature versions of the three paintings on the correct spots in the drawing of the museum (see Figure 1). The three paintings were freely dragged-and-dropped to the desired spot with a mouse placed on a platform over the handrail. When participants were satisfied with the positions of the paintings, they clicked on a "finished" icon and received performance-feedback. Hereafter, this test is referred to as the landmark test.
Next, the first-person view of the entrance appeared again and the next trial started when the participant was ready. The task was completed when participants reached the performance criterion of walking the shortest route from the entrance to the bistro twice in a row, without stopping at an intersection.

\section{Design and Procedure}

The experimental design was a 2 [topography (city block/variable)] $\times 2$ [walking demand (with support/without support)] $\times 2$ [age group (younger/older)] mixed factorial design. Topography and walking demand were manipulated within subjects and each of the four cells was filled with one maze-learning task. In the walking-demand condition with support, participants were required to hold on to the handrail during walking. In the walking-demand condition without support, participants were not allowed to hold on the handrail. The order of the conditions and the maze in each condition were rotated between individuals within groups in order to achieve a complete counterbalancing table with 16 different cells.

The experiment consisted of six different sessions. The first session involved collection of background data and administration of psychometric tests. The second session was devoted to extensive familiarization with walking on the treadmill and with the maze-learning task. Session III to VI were devoted to one experimental condition each. The individual sessions were separated by a minimum of one day and a maximum of five days. A one-way ANOVA revealed no significant age differences in the average number of days between the sessions $(F<1)$.

Session I. After signing an informed-consent form, participants were interviewed and tested for a number of background variables. After the general demographic questionnaire, several psychometric tests (reported in Participants) and a test of painting knowledge were administered. The painting knowledge test involved sorting pictures of the paintings used as experimental materials ( 3 paintings times five artists) in five piles, each labeled with the names of one of the five artists. The pictures were mounted on index cards and intermixed at the start of each trial. After each trial, the experimenter showed the correct solution. The participant repeated the sorting task until performance was perfect. To address potential age differences in preexperimental familiarity with the paintings, we performed a one-way (age group) ANOVA on the number of errors committed in the first trial. This analysis revealed no significant differences $(F<1)$. Finally, participants went through tests of global physical functioning and visual acuity.

Session II. This session started with a phase of familiarization with the treadmill, continued with familiarization with navigating in the practice maze, and ended with experimenter-supported practice on the mazelearning task. At all times when the participant was standing or walking on the treadmill, the experimenter stood next to the participant and was ready to support the participant's posture, stop the treadmill with the emergency stop, or both. If the participant entered a safety zone close to the back end of the treadmill, a safety cord, attached to the body of the participant, activated the emergency stop. These safety measures were very rarely needed and in all instances they were put into action well before any loss of balance may have occurred. If such a safety event occurred, the virtual world automatically froze and the participant restarted walking in the virtual environment from that position again.

During the familiarization phase with the treadmill, the participant walked without any virtual environment on display until expressing com-

${ }^{1}$ Pilot data showed large inter- and intraindividual differences in stopping behavior (sometimes individuals remained standing for very long periods of time) at the intersections in the mazes, thereby compromising comparisons across conditions and individuals. Therefore, the criteria for a perfect trial included both walking the shortest route and no stopping at intersections. 
fort with the equipment and the maximum speed of $3.0 \mathrm{~km} / \mathrm{hr}$. Next, the experimenter decelerated and accelerated the treadmill until the participant felt comfortable with these changes in speed. Finally, a 30-s baseline recording of the participant's posture during walking at $3.0 \mathrm{~km} / \mathrm{hr}$ was collected. To optimize the quality of the posture data, participants wore shorts, bathing suits, or other tight-fitting clothes.

Next, participants were introduced to the virtual environment and the available options for controlling movement in the museum. This phase was carried out in the city-block version of the practice maze while the participant held on to the handrail. The experimenter encouraged the participant to stroll around in the museum and try out all the five options for controlling movement. This phase continued until the participant felt comfortable with the interface.

Finally, the experimenter introduced the participant to the maze-learning task. The practice maze of the variable format was used for this purpose and the participant walked without holding on to the handrail. The standardized instructions read aloud to participants before starting the task consisted of conveying: (a) that the goal of the task was to find and remember the way to the museum's bistro; (b) that the task was completed when participants walked the shortest route from the entrance to the bistro twice in row, without stopping at an intersection; (c) that it was true that stopping at an intersection (i.e., not making a choice before reaching the intersection) was allowed at all times, but that the participant should try to avoid it because a trial counted only as perfect if the shortest route to the bistro was taken without stopping; (d) that paintings appeared only on the shortest route from the entrance to the bistro, but that participants might be on the shortest route even when no painting appeared at an intersection; (e) that the paintings and the unique objects at each intersection provided important positional information; and (f) that the participant should try to remember the locations of the paintings because their memory for this information would be tested after reaching the bistro.

With the exception of experimenter-supported familiarization with the task and repetition of task instructions during navigation performance if necessary, the procedures of session II were analogous to the procedures later carried out in the subsequent experimental sessions (see Sessions $I I I-V I)$. In other words, no direct navigational help was provided and the participant continued with the task until the performance criterion was reached.
Sessions III-VI. In each of these four experimental sessions, corresponding to the four experimental conditions, one maze-learning task was completed. Before the start of each maze, participants were again familiarized with the virtual environment and the interface. This phase was carried under the identical experimental conditions as the experimental condition assigned to this session but using the practice maze. Next, the standardized instructions were read to the participant and the task started. Short breaks between trials were allowed at the participant's request. Participants' postures were recorded from the start of the task until the performance criterion was reached. After completing the last session, participants were debriefed and compensated for their participation.

\section{Results}

\section{Maze-Learning Performance}

Several dependent measures can be extracted from the mazelearning task. We focus on distance covered and number of trials needed until the performance criterion was reached. Other possible dependent variables, such as the number of decisions made in the mazes, were highly collinear with distance covered to reach criterion and revealed substantially identical results. When nothing else is stated, statistical analyses were conducted with 2 [topography (city block/variable)] $\times 2$ [walking demand (with support/without support)] $\times 2$ [age group (younger/older)] mixed ANOVAs. Topography and walking demand were within-subject factors. The alpha level was .05 in all analyses and effect sizes are reported using partial $\eta^{2}$

Distance covered to reach criterion. Means and standard errors for this variable are depicted as a function of age group and navigation condition in Figure 2. The dependent measure is expressed in meters walked on the treadmill. An inspection of Figure 2 suggests large age-related differences in favor of younger men. On average, younger men walked $397 \mathrm{~m}$ whereas older men walked $1,117 \mathrm{~m}$ to complete the task. For the younger men, less walking was required to reach the criterion in the mazes with

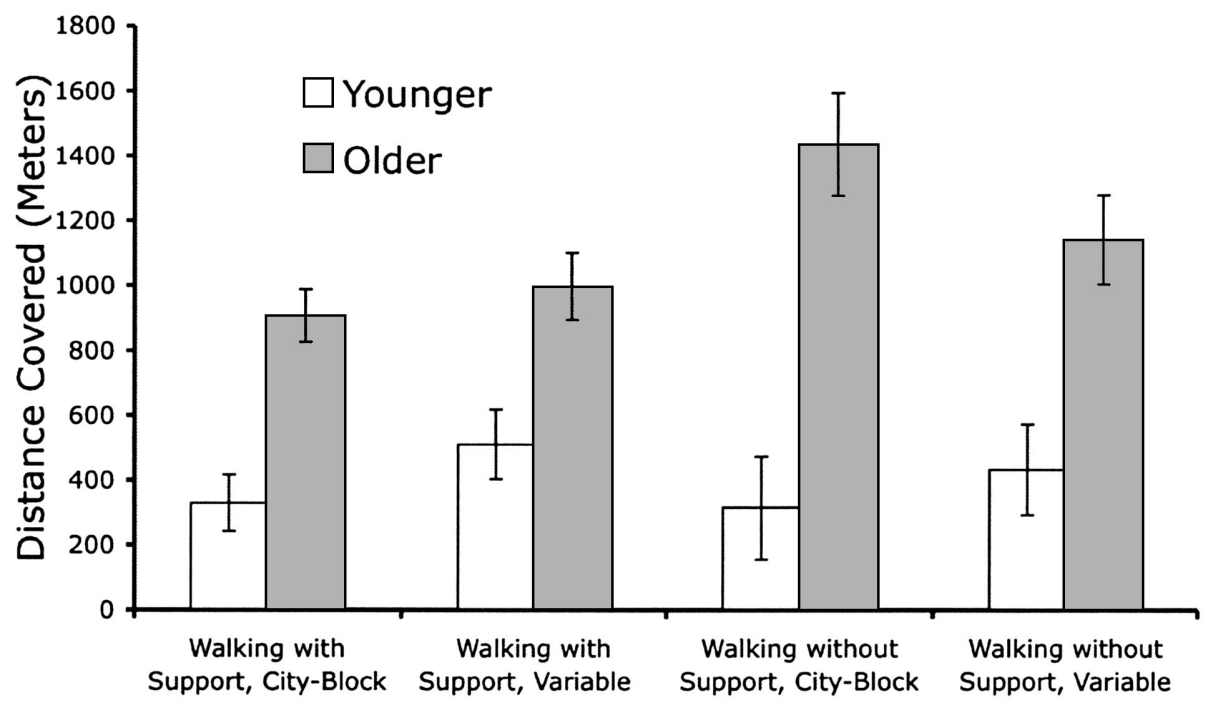

Navigation Condition

Figure 2. Mean distance covered to criterion $( \pm S E)$ as a function of age group, topography, and walking demand. 
city-block topography $(M=322)$ than in the mazes with variable topography $(M=472)$ but this difference was not apparent for the older group. It is important to note that age-related differences were reduced in the condition of walking with support $\left(M_{\text {age difference }}=528\right)$ relative to the conditions involving no support $\left(M_{\text {age difference }}=913\right)$.

The ANOVA confirmed the above observations: The main effect of age group was significant, $F(1,30)=41.19, M S E=$ 403170.42, $p<.001, \eta^{2}=.58$, there was a trend toward an age group by topography interaction, $F(1,30)=3.55, M S E=$ $141806.84, p<.07, \eta^{2}=.11$, and the age group by walking demand interaction was significant, $F(1,30)=4.15, M S E=$ 286226.18, $p<.05, \eta^{2}=.12$.

Number of trials to reach criterion. Means and standard errors for the number of trials needed to reach criterion are displayed as a function of age group and navigation condition in Figure 3. An inspection of this figure suggests that older men needed more trials to complete the task $(M=6.2)$ than younger men $(M=3.6)$. The hypothesized effect of topography is absent in the older age group, but younger men needed more trials to complete the task in the mazes of variable topography $(M=4.0)$ than in the mazes of city-block topography $(M=3.2)$. Age-related differences were reduced in the conditions involving walking support ( $\left.M_{\text {age difference }}=1.9\right)$ relative to the conditions involving no support $\left(M_{\text {age difference }}=3.2\right)$.

The statistical analyses confirmed these observations: The main effect of age group, $F(1,30)=78.90, M S E=2.63, p<.001$, $\eta^{2}=.72$, and the age group by topography interaction, $F(1,30)=$ $7.65, M S E=1.72, p<.01, \eta^{2}=.20$, were significant. There was a trend toward an age group by walking demand interaction, $F(1$, $30)=2.91, M S E=4.51, p<.10, \eta^{2}=.09$.

Landmark test performance. The dependent variable for this test was the log-transformed sum of the linear distances from the participant's placement of the paintings to the correct placements. We focused on performance in the last trial because we were interested in participants' mental representations of the positions of the paintings after performance differences in navigation were controlled for (i.e., in a trial where all participants could navigate perfectly to the goal in the maze). An inspection of the means and standard errors depicted as a function of age group and navigation condition in Figure 4 suggests that, on average, younger men $(M=$ 2.22) performed better than older men $(M=2.37)$. The analyses corroborated this observation: The main effect of age group was significant, $F(1,30)=2.27, M S E=0.09, p<.02, \eta^{2}=.20$. No other effects were significant.

\section{Posture Analyses}

Preliminary analyses. For the posture analyses, we randomly selected 30-s portions of data from the baseline posture recording (no virtual environment and walking without support) and from the second trial of each experimental condition, but with the constraint that no standing still occurred within the interval. In two cases, data of high quality were missing for the second trial and therefore replaced with data from the third or fourth trials. After deleting artifacts, linear interpolation of missing data, and T4253H smoothing (a compound data smoother), we used the two shoulder senders and the two hip senders to compute trunk angle. We focus on the trunk angle in the coronal plane only, because the corresponding measure in the sagittal plane was highly sensitivity to the frequent voluntary posture shifts in the conditions involving walking support.

Next, we computed ranges and standard deviations of trunk angles for each of the four experimental conditions. Owing to poor data quality emanating from technical problems, five participants (one younger and four older adults) were deleted because they had missing values in at least two of the four conditions. For five additional cases (two younger and three older adults), data in one of the conditions were imputed via regression from the other available variables. This imputation procedure did not substan-

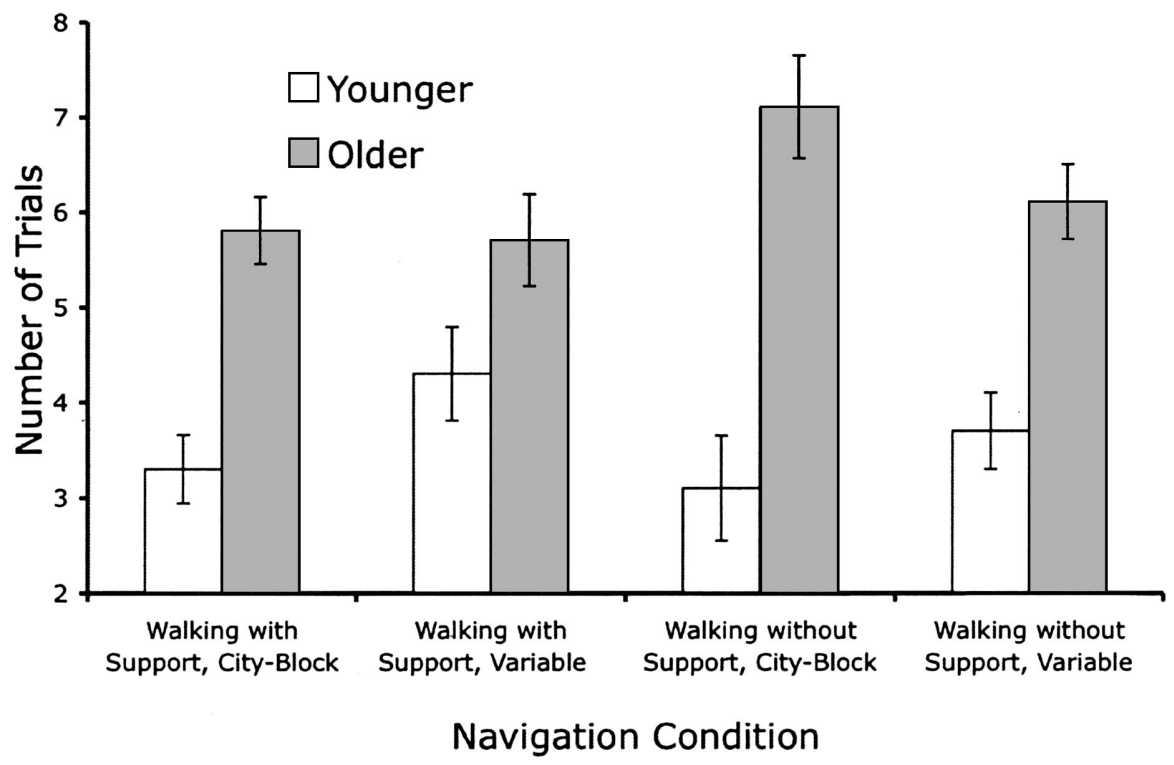

Figure 3. Mean number of trial $( \pm S E)$ as a function of age group, topography, and walking demand. 


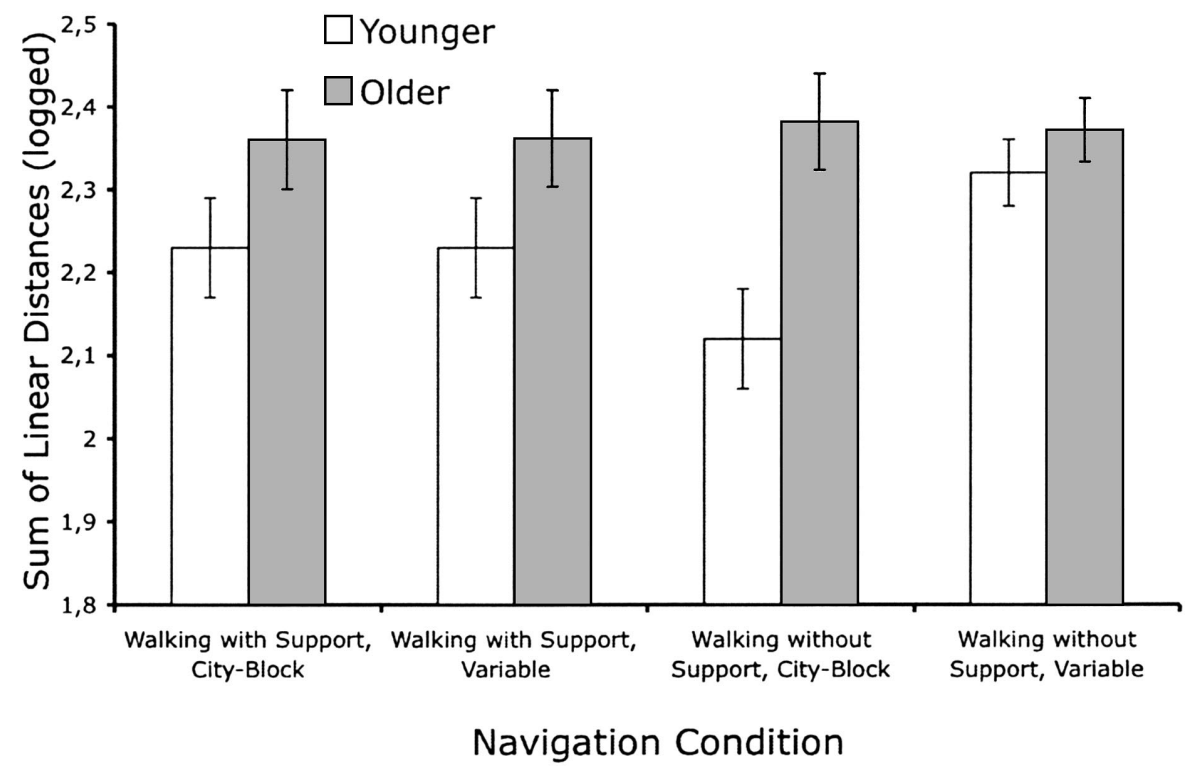

Figure 4. Mean log-transformed linear distance from correct placement $( \pm S E)$ as a function of age group, topography, and walking demand.

tively alter the mean pattern of results provided by individuals with complete data.

Trunk angle variability: Percentage increase from baseline. To control for potential baseline differences among the groups in trunk-angle variability, we computed the percentage increase from baseline [that is, (experimental condition-baseline)/baseline] for each individual and each navigation condition. Figure 5 displays the means and standard errors for ranges as a function of age group and navigation condition. An examination of this figure suggests that the conditions involving walking with support were dominated by decreases or no differences in trunk-angle variability for both younger and the older $(M=-10.6 \%)$ men. Note that the baseline condition against which these percentages are computed was collected while walking without support, which may explain the tendency for decreases observed in the condition of walking with support. In contrast, the conditions involving walking without

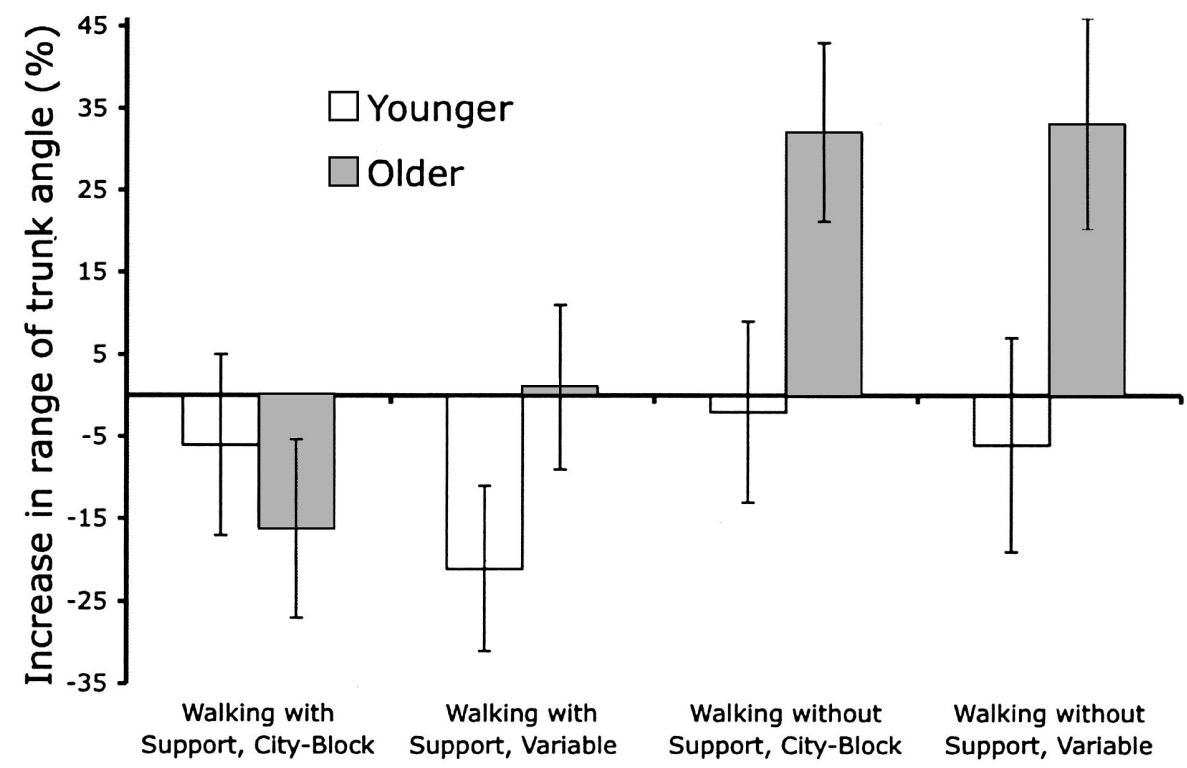

Navigation Condition

Figure 5. Mean percentage increase from baseline in range of trunk angle in the coronal plane $( \pm S E)$ as a function of age group, topography, and walking demand. 
support were dominated by increases in trunk variability for the older $(M=32.6 \%)$ but not for younger men $(-4.2 \%)$. In other words, the navigation demand increased trunk variability for elderly adults as compared to the baseline condition involving no virtual navigation task. The statistical analyses confirmed these observations: The main effect of walking demand was significant, $F(1,25)=14.28, M S E=1147.53, p<.001, \eta^{2}=.36$, but this main effect was qualified by the significant age group by walking demand interaction, $F(1,25)=5.64, M S E=1147.53, p<.03$, $\eta^{2}=.18$. The corresponding analyses for the standard deviation yielded substantially identical results: walking demand, $F(1,25)=$ 22.89, MSE $=606.41, p<.001, \eta^{2}=.48$; age group by walking, $F(1,25)=5.11, M S E=606.41, p<.04, \eta^{2}=.17$.

\section{Discussion}

This study revealed age-related differences in navigational place learning that were attenuated by providing walking support. Trunk angle variability increased more for older than for younger men while navigating without walking support. For younger, but less so for older men, navigational learning was easier in a city-block than in a variable topography. Age differences in spatial configural knowledge persisted even after all participants, young and old, had received sufficient practice to navigate to the goal without error. We discuss these findings in turn.

The age differences in navigational place learning performance can be understood and explained on many levels. At the behavioral level, this study shows that older adults' declining sensorimotor functions augment their difficulties in navigation. In other words, the age by walking demand interaction for maze-learning performance, showing greater effects of walking demand for elderly men relative to younger men, indicate that, for older adults, the sensorimotor demands of walking interact with navigation performance in a negative fashion. This finding is in line with previous studies indicating that walking per se is an attention demanding activity for elderly adults (K. Z. H. Li et al., 2001; Lindenberger et al., 2000; Lundin-Olsson et al., 1997) and extends these findings into the domain of spatial navigation. However, it is also possible that the walking-support manipulation affected spatial processes in a more direct manner. For example, elderly men in this study displayed a larger increase in trunk-angle variability than younger men in the conditions of walking without support only. It is possible that this increase in variability was accompanied by an increase in head movements that in turn might have altered vestibular signals. Considering that vestibular information might influence the acquisition of spatial representations (e.g., Stackman et al., 2002), it is possible that differences in vestibular information might have contributed to the age by walking support interaction. However, there is broad generality of the findings, ranging from clinical observations (Lundin-Olsson et al., 1997) to experimental findings from several different paradigms (see K. Z. H. Li \& Lindenberger, 2002; Woollacott \& Shumway-Cook, 2002, for reviews), suggesting age-related increase in the attentional demands of postural control. Thus, we think that the present findings highlights a general developmental quandary of behavioral aging (Lindenberger et al., 2000) in the domain of spatial navigation: While cognitive mechanisms involved in navigational place learning (e.g., attentional control, working memory) are declining with advancing age, these mechanisms simultaneously become more and more vital for avoiding the potentially harmful consequences of declining sensorimotor functions.

In this study, the age-related increase in the coupling between cognitive resources and sensorimotor functions also affected walking behavior: Relative to only walking on the treadmill (without support), indicators of trunk angle variability increased more for older than for younger adults while navigating without walking support. Under the assumption that effects of navigation load on trunk angle variability can be construed as an indicator of altered walking stability, we are tempted to suggest that the very act of navigating appeared to destabilize the gait patterns of older men. However, walking stability is not a construct that is straightforward to measure. For example, several other possible changes in the gait pattern (e.g., cadence) might have mediated the observed effects on trunk angle variability. Nevertheless, we think that it is very difficult to imagine that increases in trunk angle variability (sway) might signal increased walking stability. Thus, older men's differentially higher navigational place finding costs when walking without support cannot be portrayed as an indirect consequence of increased walking stability.

At first glance, older men's increase in trunk angle variability during navigation place learning seems inconsistent with earlier findings reporting older adults to protect walking at the expense of cognitive performance (K. Z. H. Li et al., 2001). However, this discrepancy in findings may in part reflect differences in experimental procedures. In the present experiment, sensorimotor assessments were recorded at a fixed, experimenter-defined speed of 3.0 $\mathrm{km} / \mathrm{hr}$. In contrast, in the K. Z. H. Li et al. (2001) experiment, participants were walking on a track with obstacles and not on a treadmill, and could inadvertently or deliberately adjust their walking speed while memorizing list of words.

At the information-processing level, the observed age-related impairments in navigational place learning may reflect age-related differences in multiple processes such as attentional control, spatial and relational computations in working memory, and encoding and retrieving long-term memories (see Craik \& Salthouse, 2000, for reviews). Research with animal models has highlighted the role played by associative binding deficits for older adults' impairments in spatial learning (e.g., Barnes, Suster, Shen, \& McNaughton, 1997; Tanila et al., 1997). In addition to quantitative differences, animal research suggests that aged organisms more often adopt stimulus-response learning and cue guidance strategies to locate places in space, whereas younger organisms rely more on spatial relational learning in tasks where both activities have adaptive utility (Barnes et al., 1980; Rapp et al., 1987; Tanila et al., 1997). This study provides analogues findings for humans. Younger men's' navigation performance was affected by topography to a greater degree than older men's' performance, resulting in reduced age differences in performance when navigating in the variable topography. Note that the information supporting route learning was equivalent across the two topography versions (i.e., the sequence of landmarks and left/right/straight responses supporting e.g., verbal serial learning). However, the continuous shifts in heading in the variable topography presumably placed greater demands on maintaining large-scale spatial relations among landmarks than the city-block topography. Thus, the greater effect of topography for younger age group suggests that younger men rely more on spatial navigational learning than older men. This conclusion is corroborated by the results from the landmark test, 
which demonstrate that spatial relations were acquired less well by older men than by younger men though all participants had learned to perform the navigational place finding task without error.

Given the importance of vestibular and proprioceptive cues for developing and consolidating spatial representations (e.g., Stackman et al., 2002; Waller et al., 2004), the paradigm developed for this study constitutes advancement over previous VE paradigms. At the same time, there are several limitations to the present paradigm. For example, the interface is not totally mimicking normal walking because the speed of walking is beyond the participants' control. Moreover, actions (button presses) that change directions in the VE are clearly artificial. In addition, the body-based information available in the present paradigm is limited because shifts in heading and rotations in the VE, emanating from the optic flow that specifies direction of self-motion, are mismatched with the actual constant direction of movement in space. This mismatch is potentially serious in light of evidence suggesting that important functions of vestibular and proprioceptive cues are to update heading (Klatzky, Loomis, Beall, Chance, $\&$ Golledge, 1998). It remains to be seen whether the body-based information available in the present VE task is beneficial for spatial navigation performance.

Another limitation of this study, the exclusive focus on men's' navigation performance, deserves to be highlighted. Sex differences in both quantitative (in favor of men; e.g., Sandstrom et al., 1998) and qualitative (e.g., Moffat et al., 1998) aspects of navigation performance as well sex differences in the neuroanatomical substrates of navigation behavior (Grön et al., 2000) are consistently found. In line with these findings, our experiences with the present version of paradigm include that it was difficult for many elderly women to reach the learning criterion within a session. At the same time, reductions of the difficulty level would have brought younger adults performance to ceiling. To avoid extensive dropout and associated selectivity effects, we therefore decided to focus on men only for this study. However, it is not unreasonable to predict that qualitative and quantitative sex differences in navigation performance interact with the effects reported here. Thus, it remains to be seen whether the present pattern of findings generalize to age differences between younger and older women.

To conclude, this study revealed pronounced age-related differences in navigational place learning that interacted with the demands of environmental topography on cognitive processes, indicating age-related differences in reliance on spatial relational learning. In addition, providing elderly men with walking support attenuated age-related differences in navigational place learning. These findings have important implications. For example, the interaction between sensorimotor functions and spatial navigation observed in the present study suggests that navigation aids carefully designed to fit older adults navigational activities might actually improve walking stability. Interactions between sensorimotor and cognitive dimensions of behavior, as well as qualitative age differences in navigational activities, need to be considered when trying to understand and take the edge off age-related decline in spatial navigation.

\section{References}

Aguirre, G. K., \& D’Esposito, M. D. (1999). Topographical disorientation: A synthesis and taxonomy. Brain, 122, 1613-1628.
Barnes, C. A., Nadel, L., \& Honig, W. K. (1980). Spatial memory deficits in senescent rats. Canadian Journal of Psychology, 34, 29-39.

Barnes, C. A., Rao, G., Foster, T. C., \& McNaughton, B. L. (1992). Region-specific age effect on AMPA sensitivity: Electrophysiological evidence for loss of synaptic contacts in the hippocampal field CA1. Hippocampus, 2, 457-468.

Barnes, C. A., Suster, M. S., Shen, J., \& McNaughton, B. L. (1997), Multistability of cognitive maps in the hippocampus of old rats. Nature, 388, 272-275.

Bohbot, V. D., Iaria, G., \& Petrides, M. (2004). Hippocampal function and spatial memory: Evidence from functional neuroimaging in healthy participants and performance of patients with medial temporal lobe resections. Neuropsychology, 18, 418-425.

Brauer, S. G., Woollacott, M., \& Shumway-Cook, A. (2001). The interacting effects of cognitive demand and recovery of postural stability in balance-impaired elderly persons. Journal of Gerontology: Medical Sciences, 56, 489-496.

Brown, L. A., Shumway-Cook, A., \& Woollacott, M. H. (1999). Attentional demands and postural recovery: The effects of aging. Journal of Gerontology: Medical Sciences, 54, 165-171.

Burgess, N., Maguire, E., \& O'Keefe, J. (2002). The human hippocampus and spatial and episodic memory. Neuron, 35, 625-641.

Chalfonte, B. L., \& Johnson, M. K. (1996). Feature memory and binding in young and old adults. Memory \& Cognition, 24, 403-416.

Chen, H.-C., Schultz, A. B., Ashton-Miller, J. A., Giordani, B., Alexander, N. B., \& Guire, K. E. (1996). Stepping over obstacles: Dividing attention impairs performance of old more than young adults. Journal of Gerontology: Medical Sciences, 51, 116-122.

Cohen, N. J., \& Eichenbaum, H. (1993). Memory, amnesia, and the hippocampal system. Cambridge, MA: MIT Press.

Craik, F. I. M., \& Salthouse, T. A. (2000). Handbook of aging and cognition. Mahwah, NJ: Erlbaum.

Eichenbaum, H., Dudchenko, P., Wood, E., Shapiro, M., \& Tanila, H. (1999). The hippocampus, memory, and place cells: Is it spatial memory or memory space? Neuron, 23, 209-226.

Ekstrom, A. D., Kahana, M. J., Caplan, J. B., Fields, T. A., Isham, E. A., Newman, E. L., \& Fried, I. (2003). Cellular networks underlying human spatial navigation. Nature, 425, 184-187.

Grön, G., Wunderlich, A. P., Spitzer, M., Tomczak, R., \& Riepe, M. W. (2000)., Brain activation during human navigation: Gender differential neural networks as substrates of performance. Nature Neuroscience, 3, 404-408.

Hartley, T., Maguire, E. A., Spiers, H. J., Burgess, N. (2003). The wellworn route and the path less traveled: Distinct neural bases of route following and wayfinding in humans. Neuron, 37, 877-888.

Hertzog, C., \& Rypma, B. (1991). Age differences in components of mental-rotation task performance. Bulletin of the Psychonomic Society, 29, 209-212.

Jacoby, L. L. (1999). Ironic effects of repetition: Measuring age-related differences in memory. Journal of Experimental Psychology: Learning, Memory, and Cognition, 25, 3-22.

Janzen, G., \& van Turennout, M. 82004). Selective neural representation of objects relevant for navigation. Nature Neuroscience, 7, 673-677.

Jenkins, L., Myerson, J., Joerding, J. A., \& Hale, S. (2000). Converging evidence that visuospatial cognition is more age-sensitive than verbal cognition. Psychology and Aging, 15, 157-175.

Ketcham, C. J., \& Stelmach, G. E. (2001). Age-related declines in motor control. In J. E. Birren \& K. W. Schaie (Eds.), Handbook of the psychology of aging (5th ed., pp. 349-379). San Diego, CA: Academic Press.

Kirasic, K. C. (1991). Spatial cognition and behavior in young and elderly adults: Implications for learning new environments. Psychology and Aging, 6, 10-18. 
Kirasic, K. C. (2000). Age differences in adults' spatial abilities, learning environmental layout, and wayfinding behavior. Spatial Cognition and Computation, 2, 117-134.

Klatzky, R. L., Loomis, J. M., Beall, A. C., Chance, S. S., \& Golledge, R. G. (1998). Spatial updating of self-position and orientation during real, imagined, and virtual locomotion. Psychological Science, 9, 293298.

Klein. D. A., Steinberg, M., Galik, E., Steele, C., Sheppard, J-M., Warren, A., Rosenblatt, A., \& Lyketsos, C. G. (1999). Wandering behaviour in community-residing persons with dementia. International Journal of Geriatric Psychiatry, 14, 272-279.

Laurance, H. E., Thomas, K. G. F., Newman, M. C., Kaszniak, A. W., Nadel, L., \& Jacobs, W. J. (2002). Older adults map novel environments but do not place learn: Findings from a computerized spatial task. Aging, Neuropsychology, and Cognition, 9, 85-97.

Li, K. H. Z., \& Lindenberger, U. (2002). Relation between aging sensory/ sensorimotor and cognitive functions. Neuroscience and Biobehavioral Reviews, 26, 777-783.

Li, K. Z. H., Lindenberger, U., Freund, A. M., \& Baltes, P. B. (2001). Walking while memorizing: Age-related differences in compensatory behavior. Psychological Science, 12, 230-237.

Li, S-C., Naveh-Benjamin, M., \& Lindenberger, U. (in press). Aging neuromodulation impairs associative binding: A neurocomputational account. Psychological Science.

Light, L. L., \& Zelinski, E. M. (1983). Memory for spatial information in young and old adults. Developmental Psychology, 19, 901-906.

Lindenberger, U., Marsiske, M., \& Baltes, P. B. (2000). Memorizing while walking: Increase in dual costs from young adulthood to old age. Psychology and Aging, 3, 417-436.

Lindenberger, U., Mayr, U., \& Kliegl, R. (1993). Speed and intelligence in old age. Psychology and Aging, 8, 207-220.

Lipman, P. D. (1991). Age and exposure differences in the acquisition of route information. Psychology and Aging, 6, 128-133.

Lövdén, M., Bergman, L., Adolfsson, R., Lindenberger, U., \& Nilsson, L.-G. (2005). Studying individual aging in an interindividual context: Typical paths of age-related, dementia-related, and mortality-related cognitive development in old age. Psychology and Aging, 20, 303-316.

Lövdén, M., Ghisletta, P., \& Lindenberger, U. (2004). Cognition in the Berlin Aging Study (BASE): The first ten years. Aging, Neuropsychology, \& Cognition, 11, 104-133.

Lövdén, M., \& Lindenberger, U. (2005). Development of intellectual abilities in old age: From age gradients to individuals. In O. Wilhelm and R. W. Engle (Eds.). Understanding and measuring intelligence (pp. 203-221). Thousand Oaks, CA: Sage.

Lundin-Olsson, L., Nyberg, L., \& Gustafsson, Y. (1997). Attention, frailty, and falls: The effect of a manual task on basic mobility. Journal of the American Geriatric Society, 46, 758-761.

Lundin-Olsson, L., Nyberg, L., \& Gustafsson, Y. (1998). "Stops when talking" as a predictor of falls in elderly people. The Lancet, 349, 617.

Maguire, E. A., Burgess, N., Donnett, J. G., Frackowiak, R. S., Frith, C. D., \& O'Keefe, J. (1998). Knowing where and getting there. A human navigation network. Science, 280, 921-924.

McDowd, J. M., \& Shaw, R. (2000). Attention and aging: A functional perspective. In F. I. M. Craik \& T. A. Salthouse (Eds.), Handbook of aging and cognition (2nd ed., pp. 221-292). Mahwah, NJ: Erlbaum.

McGibbon, C. A., \& Krebs, D. E. (2001). Age-related changes in lower trunk coordination and energy transfer during gait. Journal of Neurophysiology, 85, 1923-1931.

McNaughton, B. L., Barnes, C. A., Gerrard, J. L., Gothard, K., Jung, M., Knierim, J. J., Kudrimoti, H. S., Qin, Y., Skaggs, W. E., Suster, M., \& Weaver, K. L. (1996). Deciphering the hippocampal polyglot: The hippocampus as a path integration system. Journal of Experimental Biology, 199, 173-185.
Moffat, S. D., \& Hampson, E., \& Hatzipantelis, M. (1998). Navigation in a "virtual" maze: Sex differences and correlation with psychometric measures of spatial ability in humans. Evolution and Human Behavior, 19, 73-87.

Moffat, S. D., Zonderman, A. B., \& Resnick, S. M. (2001). Age differences in spatial memory in a virtual environment navigation task. Neurobiology of Aging, 22, 787-796.

Morris, R. G. M. (1981). Spatial localization does not require the presence of local cues. Learning and Motivation, 12, 239-260.

Naveh-Benjamin, M., Hussain, Z., Guez, J., \& Bar-on, M. (2003). Adult age differences in episodic memory: Further support for an associativedeficit hypothesis. Journal of Experimental Psychology: Learning, Memory, and Cognition, 29, 826-837.

O'Keefe, J. (1999). Do hippocampal pyramidal cells signal non-spatial as well as spatial information. Hippocampus, 9, 352-364.

O'Keefe, J., \& Dostrovsky, J. (1971). The hippocampus as a spatial map: Preliminary evidence from unit activity in the freely-moving rat. Brain Research, 34, 171-175.

O'Keefe, J., \& Nadel, L. (1978). The hippocampus as a cognitive map. Oxford: Oxford University Press.

Ossenkopp, K.-P., \& Hargreaves, E. L. (1993). Spatial learning in an enclosed eight-arm radial maze in rats with sodium arsanilate-induced labyrinthectomies. Behavioral and Neural Biology, 59, 253-257.

Peruch, P., \& Gaunet, F. (1998). Virtual environments as a promising tool for investigating human spatial cognition. Current Psychology of Cognition, 17, 881-899.

Prince, F., Corriveau, H., Hébert, R., \& Winter, D. A. (1997). Gait in the elderly. Gait and Posture, 5, 128-135.

Rapp, P. R., Rosenberg, R. A., \& Gallagher, M. (1987). An evaluation of spatial information processing in aged rats. Behavioral Neuroscience, 101, 3-12.

Richardson, A. E., Montello, D. R., \& Hegarty, M. (1999). Spatial knowledge acquisition from maps and from navigation in real and virtual environments. Memory \& Cognition, 27, 741-750.

Ruddle, R. A., Payne, S. J., \& Jones, D. M. (1997). Navigating buildings in "desk-top" virtual environments: Experimental investigations using extended navigation experience. Journal of Experimental Psychology: Applied, 3, 143-159.

Salthouse, T. A., \& Mitchell, D. R. (1989). Structural and operational capacities in integrative spatial ability. Psychology and Aging, 4, $18-25$.

Sandstrom, N. J., \& Kaufman, J., \& Huettel, S. A. (1998). Males and females use different distal cues in a virtual environment navigation task. Cognitive Brain Research, 6, 351-360.

Smith, T. D., Adams, M. M., Gallagher, M., Morrison, J. H., \& Rapp, P. R. (2000). Circuit-specific alterations in hippocampal synaptophysin immunoreactivity predict spatial learning impairment in aged rats. Journal of Neuroscience, 20, 6587-6593.

Spencer, W. D., \& Raz, N. (1995). Differential effects of aging on memory for content and context: A meta-analysis. Psychology and Aging, 10, $527-539$.

Stackman, R. W., Clark, A. S., \& Taube, J. S. (2002). Hippocampal spatial representations require vestibular input. Hippocampus, 12, 291-303.

Tanila, H., Sipilä, P., Shapiro, M., \& Eichenbaum, H. (1997). Brain aging: Impaired coding of novel environmental cues. Journal of Neuroscience, 17, 5167-5174.

Teasdale, N., Bard, C., LaRue, J., \& Fleury, M. (1993). On the cognitive penetrability of posture control. Experimental Aging Research, 19, 1-13.

Tolman, E. C. (1948). Cognitive maps in rats and men. Psychological Review, 55, 189-208.

Uttl, B., \& Graf, P. (1993). Episodic spatial memory in adulthood. Psychology and Aging, 2, 257-273. 
Vandenberg, S. G., \& Kuse, A. R. (1978). Mental rotation, a group test of three-dimensional spatial visualization. Perceptual and Motor Skills, 64, 907-914.

Waller, D., Loomis, J. M., \& Haun, D. B. M. (2004). Body-based senses enhance knowledge of directions in large-scale environments. Psychonomic Bulletin \& Review, 11, 157-163.

Wilkniss, S. M., Jones, M. G., Korol, D. L., Gold, P. E., \& Manning, C. A. (1997). Age-related differences in an ecologically based study of route learning. Psychology and Aging, 12, 372-375.

Witmer, B. G., Bailey, J. H., Knerr, B. W., Parsons, K. C. (1996). Virtual spaces and real world places: Transfer of route knowledge. International Journal of Human-Computer Studies, 45, 413-428.

Woollacott, M., \& Shumway-Cook, A. (2002). Attention and the control of posture and gait: A review of an emerging area of research. Gait and Posture, 16, 1-14.
Received November 9, 2004

Revision received June 24, 2005

Accepted July 12, 2005

\section{Low Publication Prices for APA Members and Affiliates}

Keeping you up-to-date. All APA Fellows, Members, Associates, and Student Affiliates receive-as part of their annual dues-subscriptions to the American Psychologist and APA Monitor. High School Teacher and International Affiliates receive subscriptions to the APA Monitor, and they may subscribe to the American Psychologist at a significantly reduced rate. In addition, all Members and Student Affiliates are eligible for savings of up to $60 \%$ (plus a journal credit) on all other APA journals, as well as significant discounts on subscriptions from cooperating societies and publishers (e.g., the American Association for Counseling and Development, Academic Press, and Human Sciences Press).

Essential resources. APA members and affiliates receive special rates for purchases of APA books, including the Publication Manual of the American Psychological Association, and on dozens of new topical books each year.

Other benefits of membership. Membership in APA also provides eligibility for competitive insurance plans, continuing education programs, reduced APA convention fees, and specialty divisions.

More information. Write to American Psychological Association, Membership Services, 750 First Street, NE, Washington, DC 20002-4242. 